\title{
Further synthesis and biological characterization of a series of 2-sulfonamidebenzamides as allosteric modulators of MrgX1
}

\author{
Swagat Sharma ${ }^{\mathrm{a}}$, Qi Peng ${ }^{\mathrm{b}}$, Anish K. Vadukoot ${ }^{\mathrm{a}}$, Christopher Aretz ${ }^{\mathrm{a}}$, Aaron A. Jensen ${ }^{\mathrm{a}}$, Alexander I. \\ Wallick $^{\mathrm{a}}$, Xinzhong Dong ${ }^{\mathrm{b}, \mathrm{c}}$, Corey R. Hopkins ${ }^{\mathrm{a},{ }^{*}}$ \\ a Department of Pharmaceutical Sciences, College of Pharmacy, University of Nebraska Medical Center, Omaha, NE USA \\ 68198-6125 \\ ${ }^{\mathrm{b}}$ The Solomon H. Snyder Department of Neuroscience, Center for Sensory Biology, Johns Hopkins University School of \\ Medicine, Baltimore, MD 21205 \\ ${ }^{c}$ Howard Hughes Medical Institute, Johns Hopkins University School of Medicine, Baltimore, MD 21205
}

\begin{abstract}
:
The present study describes our continued efforts in the discovery and characterization of a series of 2sulfonamidebenzamides as allosteric modulators of MrgX1. MrgX1 has been shown to be an attractive target as a non-opioid receptor for the potential treatment of chronic pain. Working from our original compound, ML382, and utilizing iterative medicinal chemistry, we have identified key halogen substituents that improve MrgX1 potency by $\sim 8$-fold. In addition, we have evaluated the compounds in Tier 1 DMPK assays and have identified key compounds that impart improved potency and microsomal stability.
\end{abstract}

Keywords: Mas-related G-protein coupled receptor, MrgX1, structure-activity relationship, allosteric agonist, 2-sulfonamidebenzamide

\section{Introduction:}

Chronic pain is a major health and economic problem worldwide with an estimated prevalence reaching epidemic levels of 20 to $25 \%$ of the population. ${ }^{1}$ In the United States, chronic pain affects over 116 million adults and costs up to $\$ 635$ billion annually in treatment and lost productivity, more than those for cancer, heart disease, and diabetes combined. ${ }^{2}$ Chronic pain, especially nerve injury induced neuropathic pain and spontaneous pain, is challenging to treat and often refractory to current pharmacotherapies. Because the 
major analgesics (e.g., opioids) bind to receptors that are widely expressed throughout the central nervous system (CNS), dose-limiting adverse effects and risks of addiction and abuse present substantial barriers to their clinical use. ${ }^{3}$ Pain sensing neurons (a.k.a nociceptors) in dorsal root ganglion (DRG) play essential role in pain transmission by detecting painful signals in the periphery such as skin and viscera. Therefore, targeting molecules specifically expressed in nociceptors may offer an opportunity for pain-selective pharmacologic interventions.

Mas-related G-protein couple receptors (Mrg receptors) are a large family of orphan receptors expressed in small diameter sensory neurons and represent a set of potential targets for pain. Mrgs (also called Mrgprs) are a family of orphan G-protein coupled receptors (GPCRs) consisting of more than 50 members in the mouse genome. ${ }^{4}$ Our previous data have shown that Mrgs including mouse MrgC11 and human MrgX1 are specifically expressed in nociceptors in DRG and constitute an endogenous anti-pain pathway. ${ }^{5}$ The expression of many Mrgs, such as mouse MrgC11 and its human homologue MrgX1, is restricted to subsets of nociceptors in DRG, but not detected in the CNS (i.e., the spinal cord and brain) or in the rest of the body. ${ }^{4,6}$ Unfortunately, previous studies on MrgX1 were hampered as it became clear that human MrgX1 has binding and pharmacological profiles distinct from the binding and pharmacological profiles of rodent $\mathrm{MrgC}$, thus making it difficult to translate studies since was it not feasible to use traditional animal models to evaluate MrgX1 ligands in mouse models. This roadblock was overcome with the generation of a transgenic mouse line where the MrgX1 gene is expressed in MrgC11-expressing DRG neurons. ${ }^{5}$ This new humanized mouse line makes is possible to examine MrgX1 modulators in animal models of pain. With this new mouse model, we were able to show that activating MrgX1 with an allosteric agonist (ML382) lessened both evoked pain and spontaneous pain after injury, without any observable side effects. ${ }^{5}$ ML382 was the first reported MrgX1 allosteric agonist and herein, we report additional structureactivity relationship (SAR) studies culminating in the discovery of a next-generation MrgX1 allosteric agonist. $^{7}$

Our original medicinal chemistry efforts that led to the identification of ML382, 1 (Figure 1) started with a high-throughput screen of the NIH Molecular Library Small-Molecule Repository (MLSMR) 
compound collection which contained $>300,000$ compounds utilizing a triple addition protocol. ${ }^{7}$ The screen identified a 2-(sulfonamido)- $N$-phenylbenzamide scaffold which showed promise as a starting point for the initial SAR campaign. Our efforts centered around the cyclopropylsulfonamide (blue) and the 2-ethoxy (green) moieties and from that ML382 was identified. ${ }^{7}$ Although this compound proved to be a beneficial tool compound, it did suffer from notable deficiencies. Namely, the compound did not possess suitable pharmacokinetic properties and, thus, was limited to intrathecal (i.t.) administration, and the overall MrgX1 potency was less than desired. ${ }^{5}$ Thus, we have been working on the discovery and characterization of additional MrgX1 allosteric agonists with improvement in these areas. The current work concentrated on the phenyl ring systems to minimize the oxidative metabolism, ring closing of the sulfonamide portion to five-membered ring systems as well as amide bioisosteres. The work culminated in the discovery of $\mathbf{8 e}$ which enhanced the potency by 8 -fold and improved the metabolic stability.
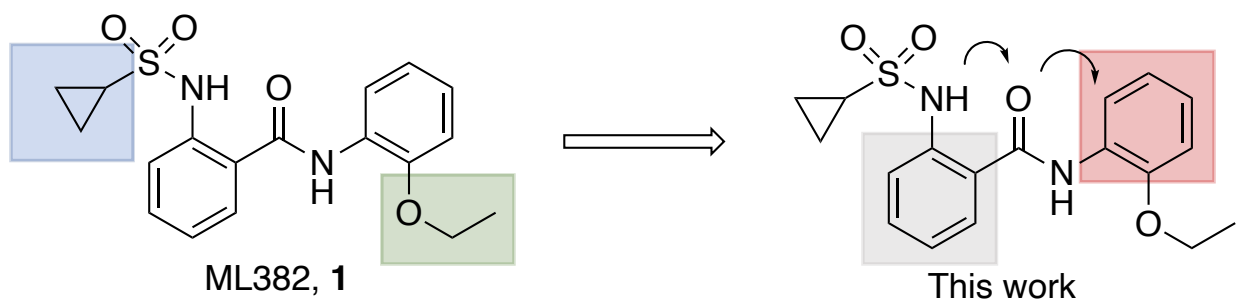

Figure 1. Initial SAR around ML382, 1 and the current work.

The synthesis of the compounds evaluated in this work is outlined in Scheme 1. The compounds that contain the main core 2 -(sulfonamido)- $N$-benzamide were all synthesized via a common route. Namely, the anthranilic acid, 2, was coupled with an appropriate sulfonyl chloride under basic water conditions $\left(\mathrm{NaHCO}_{3}, \mathrm{H}_{2} \mathrm{O}\right)$ to yield the sulfonamide, 3. This compound was then coupled with an aniline (or amine) using T3P to yield the final compounds, 4-8. ${ }^{8,9}$ This synthetic scheme was modular in that the amide could be made first and then the sulfonamide as the final target. Next, the amide bioisostere compounds were synthesized individually as outlined. The benzimidazole was synthesized by the coupling of the sulfonamide benzoic acid, 3, with 1,2-diaminophenyl via two-step protocol. First, the amide was formed 
using HATU followed by ring closure under heat and acidic conditions to yield $\mathbf{4 k} .^{10}$ The triazole was synthesized by reacting the hydrazine carbonyl, $\mathbf{1 0}$, with the cyano, $\mathbf{1 1},\left(\mathrm{K}_{2} \mathrm{CO}_{3}, \mathrm{BuOH}, 150{ }^{\circ} \mathrm{C}\right)$ to yield the desired product, $4 \mathbf{4} .^{11}$ Finally, the oxadiazole was synthesized via a two-step protocol as outlined. The hydrazine carbonyl, 13, was coupled to the acid chloride, $\mathbf{1 2}$ to yield the penultimate intermediate, $\mathbf{1 4}$, which is then cyclized under $\mathrm{POCl}_{3}$ to yield the oxadiazole, $\mathbf{4 m}$.
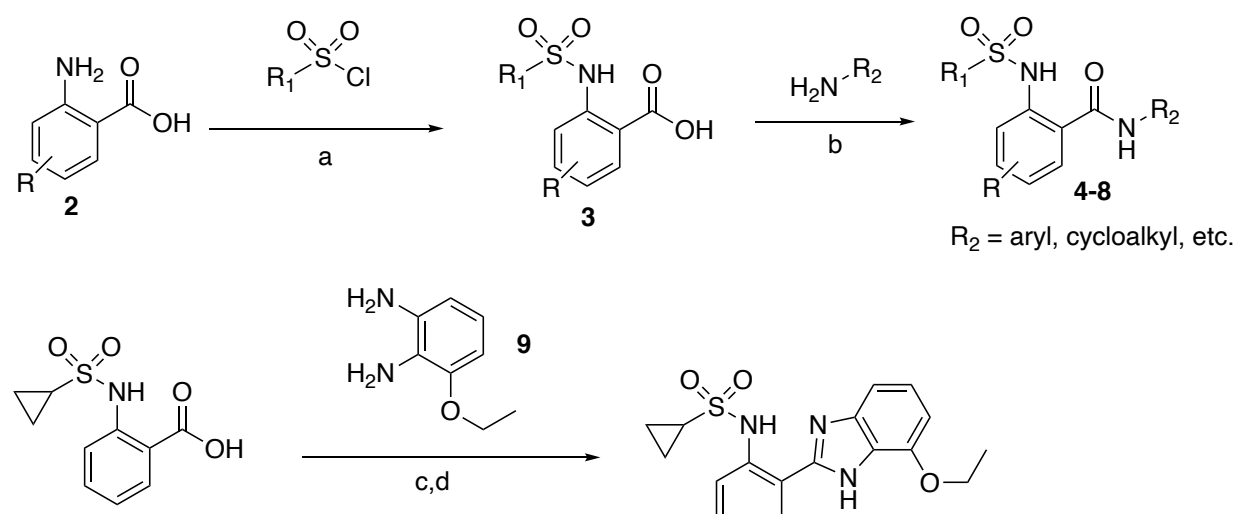

3
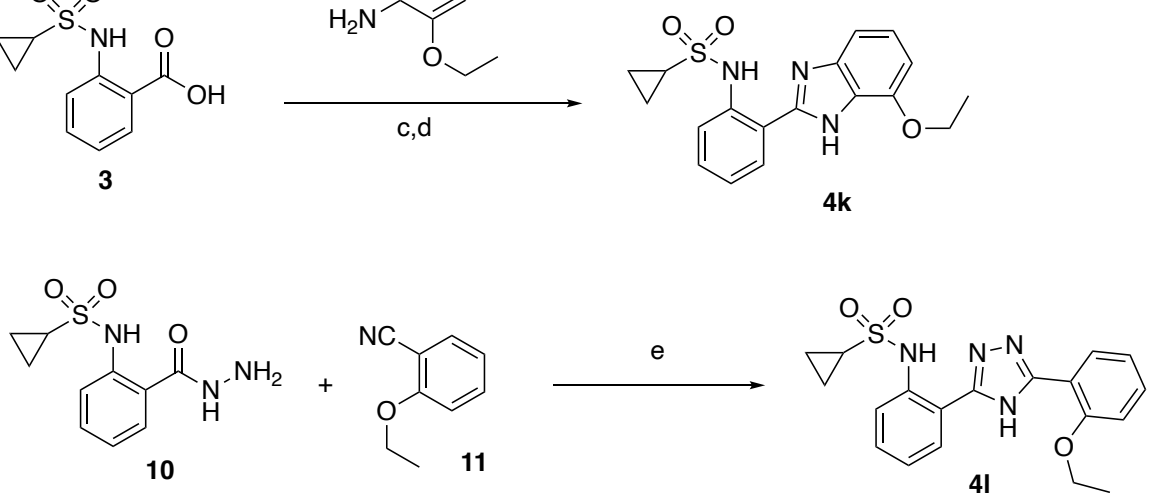

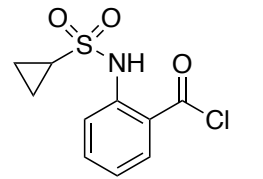

12

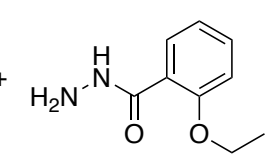

13

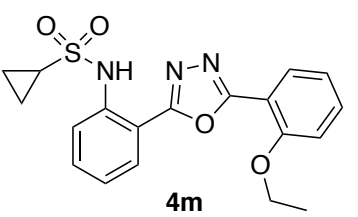

Scheme 1. Synthesis of $\mathrm{MrgX} 1$ allosteric agonists. Reagents and conditions: a) $\mathrm{NaHCO}_{3}, \mathrm{H}_{2} \mathrm{O}, \mathrm{rt}, 12 \mathrm{~h}$; b) T3P, Et ${ }_{3} \mathrm{~N}, \mathrm{CH}_{2} \mathrm{Cl}_{2}, \mathrm{rt}, 6$ h; c) HATU, iPr ${ }_{2} \mathrm{NEt}$, THF, rt, 16 h; d) AcOH, $80{ }^{\circ} \mathrm{C}, 16$ h; e) $\mathrm{K}_{2} \mathrm{CO}_{3}, \mathrm{BuOH}, 150$ ${ }^{\circ} \mathrm{C}$; f) $\left.\mathrm{Et}_{3} \mathrm{~N}, \mathrm{CH}_{2} \mathrm{Cl}_{2} ; \mathrm{g}\right) \mathrm{POCl}_{3}$ 
We envisioned closing the carbonyl of the amide with the sulfonamide to form a five-membered ring system as our first attempt to scaffold hop within this series. From our previous work, we knew the NH of the sulfonamide was not critical as alkylation of this nitrogen was tolerated. Thus, we started with commercially available indazole or aza-indazole derivatives (4a-c) (Table 1). We test the efficacy of the new compounds against MrgX1 stably expressing HEK293 cell line. The enhanced activation of MrgX1 by the compounds in the presence of agonist BAM8-22 is monitored by $\mathrm{Ca}^{2+}$ imaging assay as described before. ${ }^{7}$ Unfortunately, these compounds did not show any activity against MrgX1. In fact, nearly all of our efforts to modify the benzamide phenyl ring led to inactive compounds. These included pyridine moieties (4d-e), expanding with a naphthyl moiety (4f-g) as well as changing the 1,2-relationship of the sulfonamide and the amide portion of the molecule $(\mathbf{4 h}-\mathbf{i})$.

Moving to the amide portion of the molecule also did not provide any beneficial compounds. Attempts to alkylate the nitrogen $(\mathbf{4 j})$ or insert amide isosteres, such as benzimidazole $(\mathbf{4 k})$, triazole $(\mathbf{4 l})$ and oxadiazole (4m) were all inactive. Other efforts to remove the aryl group on the right-hand side (4n), addition of an additional methylene linker (4o) or a pyridine moiety (4p) were all unproductive changes to the core scaffold.

Table 1. Modification of the benzamide core scaffold.

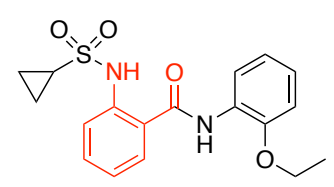

\begin{tabular}{|c|c|c|c|}
\hline Cmpd & Structure & $\mathbf{E}_{\max }(\mathbf{\%})^{\mathbf{a}}$ & $\operatorname{MrgX1}_{\mathbf{E}} \mathbf{E C}_{\mathbf{5 0}}(\boldsymbol{\mu M})^{\mathbf{a}}$ \\
\hline 1 & & 100 & 0.124 \\
\hline $\mathrm{a}$ &
\end{tabular}




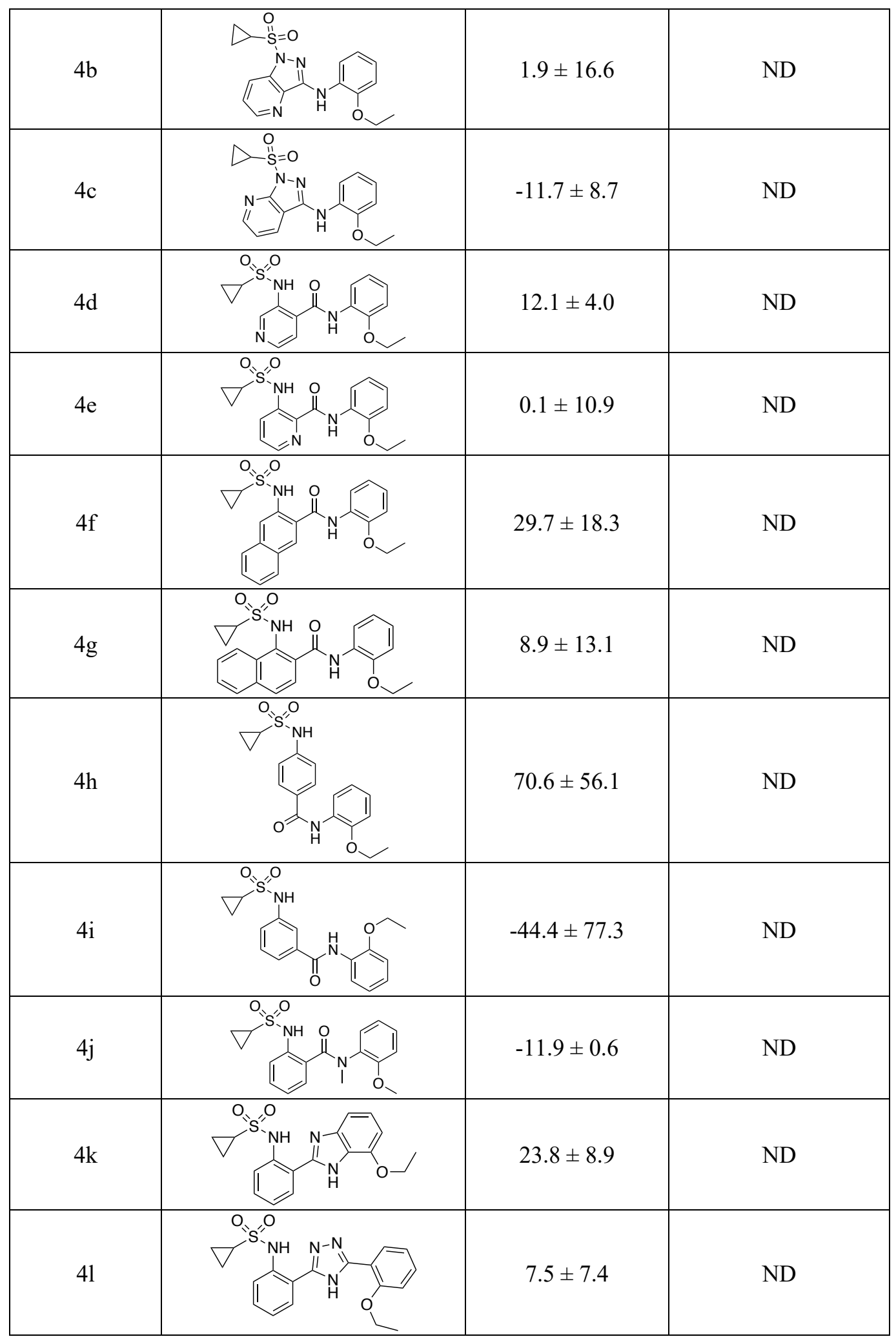




\begin{tabular}{|l|l|l|}
\hline $4 \mathrm{~m}$ & \\
\hline 40 & $12.7 \pm 13.6$ & $\mathrm{ND}$ \\
\hline $4 \mathrm{p}$ &
\end{tabular}

Having evaluated making core changes to the phenyl ring systems, we next moved to the ether moiety for modification. First, the ethyl ether was investigated by introducing branching, elongating the chain, and addition of a trifluoromethyl group. Introduction of a branched group produced active compounds; however, these lost $\sim 10$-fold activity compared to the ethyl group $\left(\mathbf{5 a}, \mathrm{EC}_{50}=2.83 \mu \mathrm{M} ; \mathbf{5 b}, \mathrm{EC}_{50}=3.14\right.$ $\mu \mathrm{M})$. Adding an additional methylene group (Propyl, 5c, $\mathrm{EC}_{50}=0.506 \mu \mathrm{M}$ ) or the 2,2,2-trifluoroethyl group $\left(\mathbf{5 d}, \mathrm{EC}_{50}=0.502 \mu \mathrm{M}\right)$ were active compounds, although not as potent as 1. Moving to the methoxyethyl (5e) lost all activity but the cyclopropylmethyl group brought some activity back to the molecule (5f, $\mathrm{EC}_{50}$ $=1.36 \mu \mathrm{M})$. Interestingly, the 2,4-diethoxy analog, $\mathbf{5 g}$, retained some potency with $\mathbf{1}\left(\mathrm{EC}_{50}=1.09 \mu \mathrm{M}\right)$; however, the 4-methoxy analog, $\mathbf{5 h}$, was inactive. Next, we moved to evaluate cyclized versions on the right-hand side of the molecule. The first analogs were cyclized via the amide nitrogen to impart rigidity into the molecule; however, these compounds, $\mathbf{5 i}$-j, were not active, which is consistent with the methylated amide analog, 4k. Additional attempts to cyclize the ether analogs were also not productive changes, 5k-q, with the lone exception of the 2-methyl-7-benzofuran analog $\mathbf{5 r}\left(\mathrm{EC}_{50}=1.04 \mu \mathrm{M}\right)$.

Table 2. Modifications of the ether moiety. 


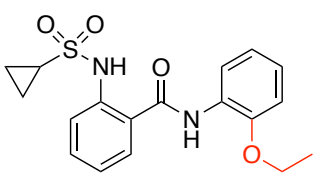

\begin{tabular}{|l|l|l|l|}
\hline Cmpd & Emax (\%) & MrgX1 EC \\
\hline (MM)
\end{tabular}




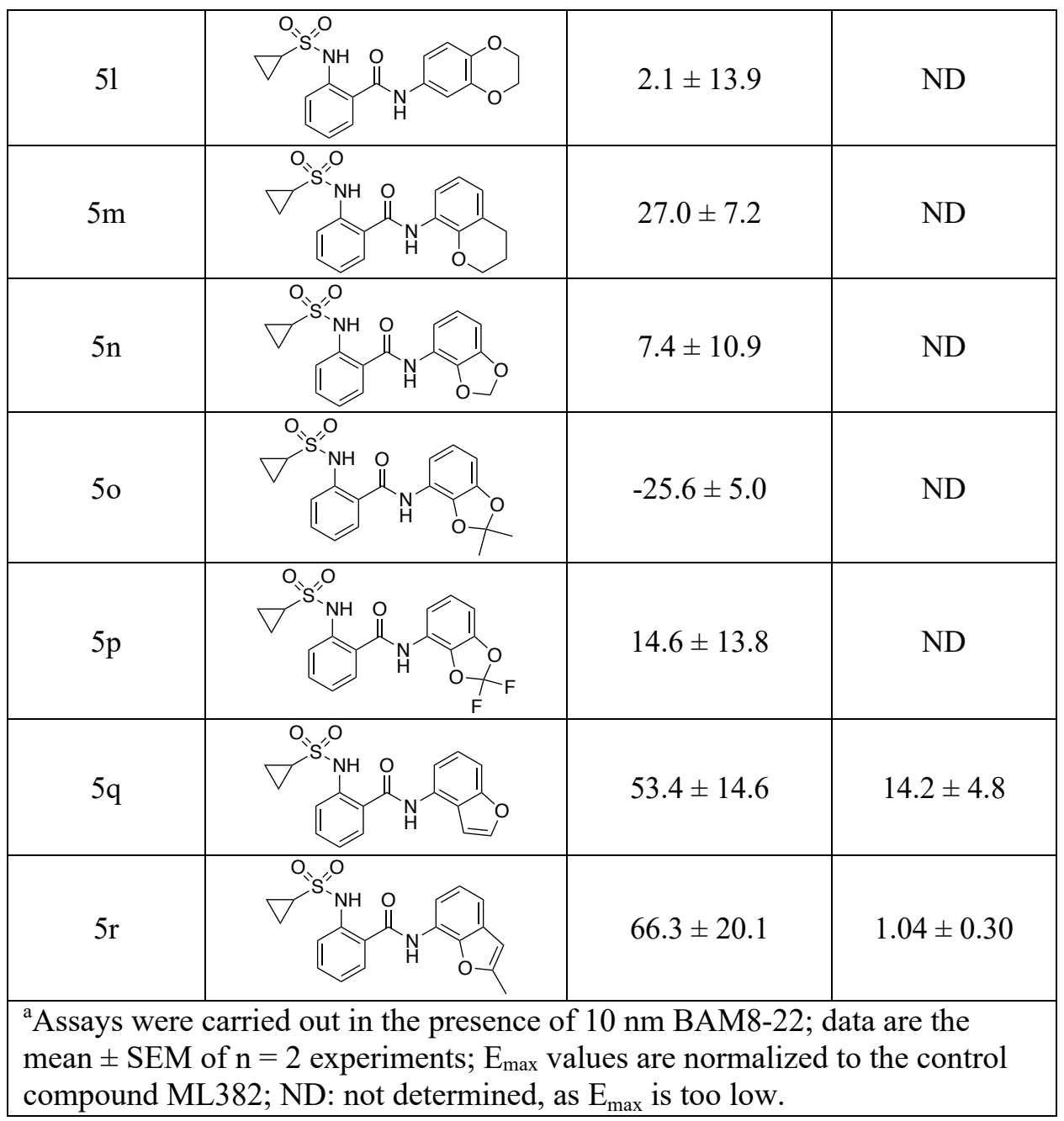

Having established the SAR around the benzamide and the ether moieties of the molecule, we next turned our attention to substitutions on the phenyl rings. From the previous SAR, the substitutions chosen were single methyl or halogen groups, as it was evident that larger modifications were not tolerated. For these studies we used both the cyclopropylsulfonamide and the ethylsulfonamide as it was shown in our previous work that these two were well tolerated. In addition, we used the ethyl and the 2,2,2-trifluoroethyl as these were also active moieties. The right-hand selections were also based on those that are commercially available as the corresponding anilines. The compounds tested for this portion of the SAR are shown in Table 3. The 4-fluoro derivatives, 6a-b, were not as active as the parent compounds; however, the 5-fluoro derivatives, $\mathbf{6 c - d}$, were very potent $\left(\mathbf{6 c}, \mathrm{EC}_{50}=0.014 \mu \mathrm{M} ; \mathbf{6 d}, \mathrm{EC}_{50}=0.285 \mu \mathrm{M}\right)$. 
Table 3. Right-hand halogen SAR.

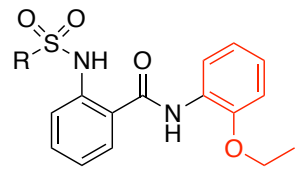

\begin{tabular}{|c|c|c|c|}
\hline Cmpd & Emax (\%) & $\begin{array}{c}\text { MrgX1 EC50 } \\
(\mu \mathrm{M})\end{array}$ \\
\hline $6 \mathrm{a}$ & & $-10.5 \pm 8.9$ & $\mathrm{ND}$ \\
\hline $6 \mathrm{~b}$ & & & \\
\hline $6 \mathrm{c}$ & & & \\
\hline
\end{tabular}

${ }^{a}$ Assays were carried out in the presence of $10 \mathrm{~nm}$ BAM8-22; data are the mean \pm SEM of $n=2$ experiments; $E_{\max }$ values are normalized to the control compound ML382; ND: not determined, as $\mathrm{E}_{\max }$ is too low.

The left-hand portion of the molecule afforded more analogs as there were more commercially available starting materials to utilize. In general, the 5-substitued analogs produced potent MrgX1 allosteric agonists (7a-I), many that were more potent than $\mathbf{1}$, and the ethoxy analogs were more potent than the 2,2,2trifluoromethyl analogs. Some of the highlighted compounds were 5-methyl, 7a $\left(\mathrm{EC}_{50}=0.104 \mu \mathrm{M}\right), \mathbf{7 g}$ $\left(\mathrm{EC}_{50}=0.098 \mu \mathrm{M}\right)$, and $7 \mathbf{i}\left(\mathrm{EC}_{50}=0.054 \mu \mathrm{M}\right)$, although nearly all of the compounds showed potency $<1$ $\mu \mathrm{M}$. The 4-substituted analogs $(\mathrm{Me}, \mathrm{F}$, or $\mathrm{Cl})$ were significantly less potent and in most cases were inactive altogether.

Table 4. Analysis of the left-hand phenyl ring system.

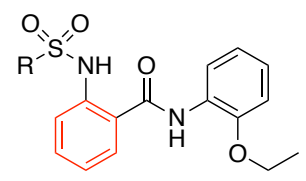




\begin{tabular}{|c|c|c|c|}
\hline Cmpd & Structure & $\operatorname{Emax}(\%)$ & $\begin{array}{c}\text { MrgX1 EC } \\
(\mu \mathrm{M})\end{array}$ \\
\hline $7 a$ & & $104.0 \pm 8.9$ & $0.103 \pm 0.027$ \\
\hline $7 b$ & & $89.9 \pm 4.0$ & $0.369 \pm 0.003$ \\
\hline $7 \mathrm{c}$ & & $108.5 \pm 2.1$ & $0.217 \pm 0.055$ \\
\hline $7 d$ & & $58.4 \pm 6.0$ & ND \\
\hline $7 \mathrm{e}$ & & $102.1 \pm 3.8$ & $0.173 \pm 0.035$ \\
\hline $7 f$ & & $59.8 \pm 5.9$ & ND \\
\hline $7 \mathrm{~g}$ & & $106.7 \pm 3.3$ & $0.098 \pm 0.028$ \\
\hline $7 \mathrm{~h}$ & & $100.4 \pm 2.6$ & $0.435 \pm 0.082$ \\
\hline $7 \mathrm{i}$ & & $110.0 \pm 3.4$ & $0.054 \pm 0.012$ \\
\hline $7 \mathrm{j}$ & & $103.4 \pm 9.1$ & $0.915 \pm 0.222$ \\
\hline
\end{tabular}




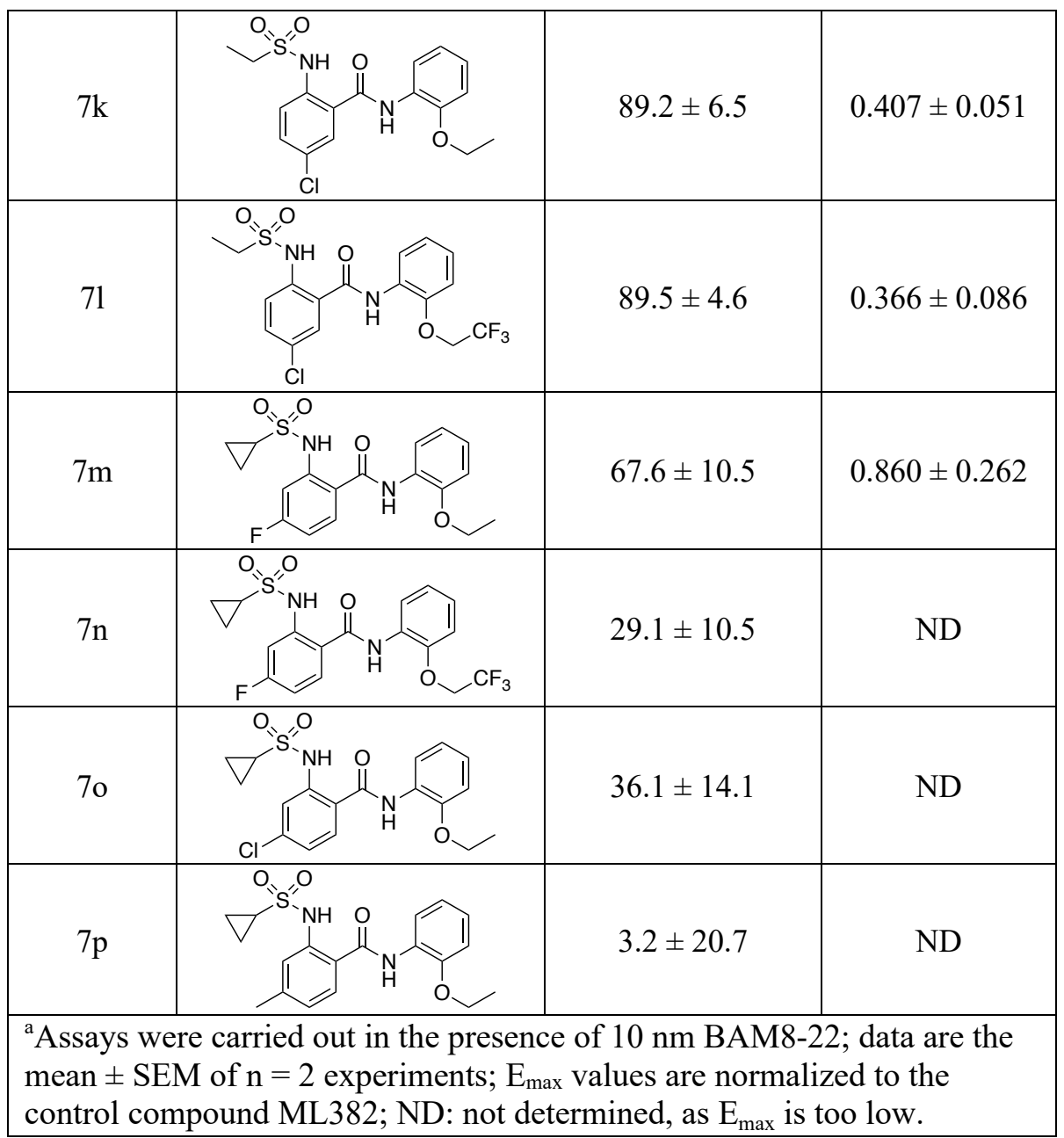

Having established the optimal substitution patterns on the individual phenyl ring systems, we next investigated the matching of the best pairs from the above study. As we only had one active right-hand substitution pattern, this was kept constant, and we varied the left-hand portion. All of the synthesized analogs were active, and the matched pairs were equipotent with the other analogs. For example, the 5chloro, 8a $\left(\mathrm{EC}_{50}=0.055 \mu \mathrm{M}\right), 5$-methyl, 8c $\left(\mathrm{EC}_{50}=0.069 \mu \mathrm{M}\right)$ and 5-fluoro, 8e $\left(\mathrm{EC}_{50}=0.013 \mu \mathrm{M}\right)$ were all of similar activity (as were the corresponding ethylsulfonamide analogs). These analogs emphasize the steric environment for the benzamide portion of the molecule as other, larger groups (naphthyl and indazole, Table 1) were all inactive, whereas the smaller substituents were tolerated. In addition to steric considerations, these analogs also show that electronic factors are not as important (electron donating vs. electron withdrawing). Overall, the SAR effort around this scaffold showed little tolerability for major 
scaffold changes, or even smaller changes around the ether substituent. This work highlights many of the known challenges in the allosteric modulator field, namely the steep and flat SAR associated with allosteric modulator drug discovery.

Table 5. SAR of the matched pairs of phenyl substituents.

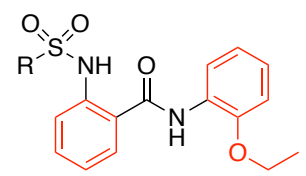

\begin{tabular}{|c|c|c|c|}
\hline Cmpd & Structure & $\operatorname{Emax}(\%)$ & $\begin{array}{c}\operatorname{MrgX1~EC}_{50} \\
(\mu \mathrm{M})\end{array}$ \\
\hline $8 a$ & & $103.5 \pm 3.6$ & $0.055 \pm 0.027$ \\
\hline $8 b$ & & $118.4 \pm 21.9$ & $0.206 \pm 0.019$ \\
\hline $8 \mathrm{c}$ & & $105.6 \pm 3.7$ & $0.069 \pm 0.024$ \\
\hline $8 \mathrm{~d}$ & & $127.6 \pm 26.8$ & $0.080 \pm 0.031$ \\
\hline $8 \mathrm{e}$ & & $126.8 \pm 25.2$ & $0.013 \pm 0.001$ \\
\hline $8 \mathrm{f}$ & & $136.3 \pm 26.8$ & $0.031 \pm 0.009$ \\
\hline \multicolumn{4}{|c|}{$\begin{array}{l}{ }^{a} \text { Assays were carried out in the presence of } 10 \mathrm{~nm} \text { BAM8-22; data are the } \\
\text { mean } \pm \text { SEM of } n=2 \text { experiments; } E_{\max } \text { values are normalized to the } \\
\text { control compound ML382; ND: not determined, as } E_{\max } \text { is too low. }\end{array}$} \\
\hline
\end{tabular}


Having identified several compounds that showed $\mathrm{EC}_{50}$ values of $<1 \mu \mathrm{M}$, we next profiled select compounds in in vitro DMPK assays to assess their human and mouse liver microsomal intrinsic clearance and plasma protein binding (Table 6). ${ }^{12,13}$ All the compounds evaluated displayed high intrinsic clearance and were predicted to have high hepatic clearance in vivo $\left(\mathrm{CL}_{\mathrm{HEP}}>75 \% \mathrm{Q}_{H}\right)$, with a few notable exceptions $(5 \mathrm{~d}, 7 \mathrm{e}, 7 \mathrm{~g})$. From a previous metabolite identification study, we anticipated that the major metabolite on 1, was oxidation of the left-hand phenyl ring. Thus, it was disappointing that the compounds that incorporated a halogen substituent on that ring did not translate to improved clearance. Although, the metabolism could shift from oxidation of the phenyl ring being the major metabolite to potentially Odealkylation in the new compounds. Using equilibrium dialysis, the plasma protein binding of the selected compounds was determined in human and mouse plasma. The results revealed that all the tested compounds were highly protein bound in human plasma $\left(\% f_{\mathrm{u}}<0.5\right)$. However, the compounds showed a better plasma protein binding profile in mouse plasma with several compounds displaying moderate free fraction ( $>2 \%)$. In addition, we profiled select compounds for selectivity against the closely related MrgX2 receptor and we tested them against the Psychoactive Drug Screening Panel at the University of North Carolina, Chapel Hill. ${ }^{14}$ This panel consists of 45 receptors and transporters that are of importance in the CNS. All the compounds tested were selective against MrgX2 (Table 6), except for $7 \mathbf{i}$.

Table 6. MrgX2 selectivity and In vitro DMPK properties of selected compounds.

\begin{tabular}{|c|c|c|c|c|c|c|c|c|}
\hline \multirow{2}{*}{ Cmpd } & \multirow{2}{*}{$\begin{array}{c}\operatorname{MrgX} 1, \\
\mathrm{EC}_{50}(\mu \mathrm{M})\end{array}$} & \multirow{2}{*}{$\begin{array}{c}\text { MrgX2, } \\
\text { \%Activation }\end{array}$} & \multicolumn{4}{|c|}{ Intrinsic Clearance $(\mathrm{mL} / \mathrm{min} / \mathrm{kg})^{\mathrm{c}}$} & \multicolumn{2}{|c|}{$\begin{array}{l}\text { Plasma Protein } \\
\text { Binding }\left(\% f_{\mathrm{u}}\right)^{\mathrm{b}}\end{array}$} \\
\hline & & & $\mathrm{hCL}_{\mathrm{INT}}$ & $\mathrm{hCL}_{\mathrm{HEP}}^{\mathrm{a}}$ & $\mathrm{mCL}_{\mathrm{INT}}$ & $\mathrm{mCL}_{\mathrm{HEP}} \mathrm{a}^{\mathrm{a}}$ & Human & Mouse \\
\hline 1 & $0.124^{\mathrm{a}}$ & $\mathrm{ND}^{\mathrm{e}}$ & 61.9 & 15.2 & 1,065 & 83.1 & 0.4 & 1.7 \\
\hline $5 \mathrm{c}$ & 0.506 & $\mathrm{ND}^{\mathrm{e}}$ & 115.6 & 17.1 & 4888.6 & 88.5 & $<0.3$ & 1.5 \\
\hline $5 \mathrm{~d}$ & 0.502 & $\mathrm{ND}^{\mathrm{e}}$ & 40.2 & 13.4 & 979.1 & 82.5 & 0.4 & 2.2 \\
\hline $5 \mathrm{r}$ & 1.04 & $\mathrm{ND}^{\mathrm{e}}$ & 74.9 & 15.8 & 1457.3 & 84.9 & $<0.3$ & 2.6 \\
\hline $6 \mathrm{~b}$ & 1.40 & $\mathrm{ND}^{\mathrm{e}}$ & 80.0 & 16.1 & 1210.7 & 83.9 & 0.5 & 2.1 \\
\hline $6 \mathrm{c}$ & 0.014 & $0.33 \pm 30.7$ & 79.1 & 16.0 & 1659 & 85.5 & 0.4 & 2.2 \\
\hline $7 \mathrm{a}$ & 0.103 & $14.4 \pm 26.5$ & 75.1 & 15.9 & 410.2 & 73.9 & 0.3 & 4.3 \\
\hline $7 \mathrm{e}$ & 0.173 & $48.5 \pm 25.9$ & 45.4 & 13.9 & 383.2 & 72.9 & 0.4 & 4.1 \\
\hline $7 \mathrm{~g}$ & 0.098 & $35.4 \pm 3.7$ & 36.0 & 12.9 & 269.0 & 67.5 & 0.2 & 4.1 \\
\hline $7 \mathrm{i}$ & 0.054 & $156.2 \pm 3.0$ & 129.2 & 17.4 & $1,470.4$ & 85.0 & 0.1 & 0.1 \\
\hline $8 a$ & 0.055 & $64.2 \pm 24.0$ & 138.4 & 17.6 & $>2,400$ & 86.8 & 0.1 & 0.3 \\
\hline $8 \mathrm{c}$ & 0.069 & $-8.8 \pm 10.9$ & 114.3 & 17.1 & $1,101.6$ & 83.3 & 0.3 & 1.2 \\
\hline $8 \mathrm{~d}$ & 0.080 & $-8.3 \pm 18.7$ & 69.3 & 15.6 & $1,004.3$ & 82.7 & 0.3 & 1.4 \\
\hline $8 \mathrm{e}$ & 0.013 & $-2.1 \pm 9.1$ & 59.4 & 15.0 & 979.5 & 82.5 & 0.2 & 1.1 \\
\hline
\end{tabular}




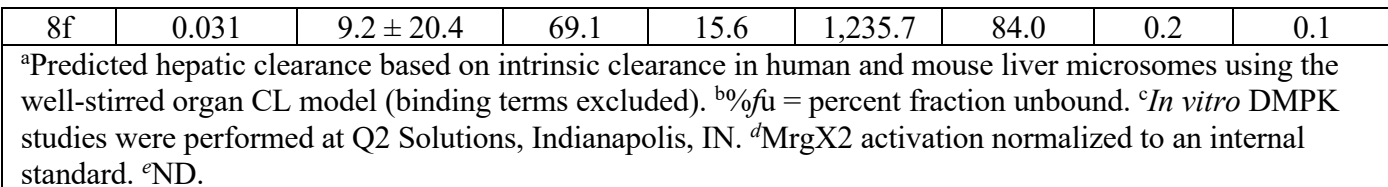

Finally, we evaluated a smaller set of selected compounds $(\mathbf{6 c}, \mathbf{8 c}, \mathbf{8 e})$ in a rat IV cassette study to assess their ability to cross the blood-brain barrier and evaluate their in vivo clearance (Table 7). ${ }^{15,16}$ The cassette study was done in a 5-in-1 format where five compounds are dosed in a single cassette (IV, $0.25 \mathrm{mg} / \mathrm{kg}$ ) and then evaluated for their plasma and brain concentrations. These compounds had varying levels of brain penetrance with $\mathbf{6 c}$ being the best $\left(\mathrm{K}_{\mathrm{p}}=0.57\right)$. However, $8 \mathbf{e}$, had the better overall plasma profile with low clearance $(\mathrm{CL}=14.1 \mathrm{ml} / \mathrm{min} / \mathrm{kg})$ and better overall plasma exposure $(\mathrm{AUC}=304 \mathrm{~h} * \mathrm{ng} / \mathrm{mL})$.

Table 7. In vivo rat cassette.

\begin{tabular}{|c|c|c|c|c|c|c|c|}
\hline \multirow[b]{2}{*}{ Cmpd } & \multicolumn{4}{|c|}{ IV Bolus $(0.25 \mathrm{mg} / \mathrm{kg})^{\mathrm{a}}$} & \multicolumn{3}{|c|}{$\begin{array}{l}\text { Plasma:Brain partitioning }(0.25 \mathrm{mg} / \mathrm{kg}, \mathrm{t} \\
=15 \mathrm{~min})^{\mathrm{a}}\end{array}$} \\
\hline & $\begin{array}{l}\mathrm{CL} \\
(\mathrm{mL} / \mathrm{min} / \mathrm{kg})\end{array}$ & $\begin{array}{l}\mathrm{T}_{1 / 2} \\
(\mathrm{~h})\end{array}$ & $\begin{array}{l}\text { AUC } \\
(\mathrm{h} * \mathrm{ng} / \mathrm{mL})\end{array}$ & Vss (L/Kg) & $\begin{array}{l}\text { Plasma } \\
\text { (ng/mL) }\end{array}$ & $\begin{array}{l}\text { Brain } \\
(\text { ng/g) }\end{array}$ & $\mathrm{K}_{\mathrm{p}}^{\mathrm{b}}$ \\
\hline 6c & 36.7 & 0.48 & 119 & 0.52 & 79.6 & 23.6 & 0.29 \\
\hline $8 c$ & 63.1 & 0.42 & 66.2 & 1.2 & 61.5 & 35.1 & 0.57 \\
\hline $8 \mathrm{e}$ & 14.1 & 0.41 & 304 & 0.34 & 297 & 55.9 & 0.19 \\
\hline \multicolumn{8}{|c|}{$\begin{array}{l}\text { In vivo DMPK studies were performed at Pharmaron Laboratories, Louisville, KY. Cassette dosing (5-in- } 1) \text { dose } \\
\text { of } 0.25 \mathrm{mg} / \mathrm{kg} \text { (IV) of each test article to male Sprague-Dawley rats. Formulation: DMSO:PEG400:EtOH:Saline } \\
(5: 48: 10: 37) \text {. Plasma blood sampling at } 0.0833,0.25,0.5,1,3,4,8 \text { and } 24 \mathrm{~h} \text { and Day } 20.25 \mathrm{~h} \text { post dose. Brain } \\
\text { sample on Day } 2 \text { at } 0.25 \mathrm{~h} .{ }^{\mathrm{b}} \mathrm{K}_{\mathrm{p}}=\text { total brain/plasma ratio. }\end{array}$} \\
\hline
\end{tabular}

In summary, we have identified additional 2-sulfonamidebenzamides as selective positive allosteric modulators of MrgX1. This work is an extension of our previous report and the compounds identified represent an $\sim 8$-fold increase in potency. The SAR highlighted the steep and flat SAR around this scaffold, a feature that is well-documented in the allosteric modulator discovery field. Compounds were also profiled in our Tier 1 DMPK assays and possess favorable plasma protein binding in mice; however, the compounds displayed high intrinsic clearance in both species tested. Selected compounds were also profiled in vivo, and they displayed moderate brain penetration; however, 8e, displayed good clearance and plasma concentrations. Due to the limitations in the PK properties, these newly identified compounds would be 
limited to non-oral dosage regimens (e.g., intraperitoneal, subcutaneous, or intrathecal); however, they do represent a major improvement in potency compared to the previously known MrgX1 allosteric agonists.

\section{Acknowledgements}

This work was generously supported by a grant from the US National Institutes of Health (NIDA: R33DA045303) to C.R.H. The authors would like to thank Q2 Solutions (Indianapolis, IN USA) for the in vitro DMPK experiments and Pharmaron (Louisville, KY) for the in vivo DMPK experiments.

\section{References}

1. Gold, M. S.; Gebhart, G. F. Nociceptor sensitization in pain pathogenesis. Nat. Med. 2010, 16, 12481257.

2. Meghani, S. H.; Polomano, R. C.; Tait, R. C.; Vallerand, A. H.; Anderson, K. O.; Gallagher, R. M. Advancing a national agenda to eliminate disparities in pain care: directions for health policy, education, practice, and research. Pain Med. 2012, 13, 5-28.

3. Raja, S. N.; Haythornthwaite, J. A. Combination therapy for neuropathic pain - which drugs, which combination, which patients? N. Eng. J. Med. 2005, 352, 1373-1375.

4. Dong, X.; Han, S.-k.; Zylka, M. J.; Simon, M. I.; Anderson, D. J. A diverse family of GPCRs expressed in specific subsets of nociceptive sensory neurons. Cell 2001, 106, 619-632.

5. Li, Z.; Tseng, P.-Y.; Tiwari, V.; Xu, Q.; He, S.-Q.; Wang, Y.; Zheng, Q.; Han, L.; Wu, Z.; Blobaum, A. L.; Cui, Y.; Tiwari, V.; Sun, S.; Cheng, Y.; Huang-Lionnet, J. H. Y.; Geng, Y.; Xiao, B.; Peng, J.; Hopkins, C.; Raja, S. N.; Guan, Y.; Dong, X. Targeting human Mas-related G protein-coupled receptor X1 to inhibit persistent pain. Proc. Natl. Acad. Sci. U. S. A. 2017, 114, E1996-E2005.

6. He, S.-Q.; Han, L.; Li, Z.; Xu, Q.; Tiwari, V.; Yang, F.; Guan, X.; Wang, Y.; Raja, S. N.; Dong, X.; Guan, Y. Temporal changes in Mrgc expression after spinal nerve injury. Neurosci. 2014, 261, 43-51.

7. Wen, W.; Wang, Y.; McManus, O. B.; Wu, M.; Li, M.; Lindsley, C. W.; Dong, X.; Hopkins, C. R. Discovery and characterization of 2-(cyclopropanesulfonamido)- $N$-(2-ethoxyphenyl)benzamide, 
ML382: a potent and selective positive allosteric modulator of MrgX1. ChemMedChem 2015, 10, 5761.

8. Dunetz, J. R.; Xiang, Y.; Baldwin, A.; Ringling, J. General and scalable amide bond formation with epimerization-prone substrates using T3P and pyridine. Org. Lett. 2011, 13, 5048-5051.

9. Garcia, A. L. L. T3P: a convenient and useful reagent in organic synthesis. Synlett 2007, 8, 1328-1329.

10. Zhu, G.-D.; Gong, J.; Gandhi, V. B.; Liu, X.; Shi, Y.; Johnson, E. F.; Donawho, C. K.; Ellis, P. A.; Bouska, J. J.; Ostrling, D. J.; Olson, A. M.; Park, C.; Luo, Y.; Shoemaker, A.; Giranda, V. L.; Penning, T. D. Discovery and SAR of orally efficacious tetrahydropyridopyridazinone PARP inhibitors for the treatment of cancer. Bioorg. Med. Chem. 2012, 20, 4635-4645.

11. Potts, K. T. The chemistry of 1,2,4-triazoles. Chem. Rev. 1961, 61, 87-127.

12. Obach, R. S. Prediction of human clearance of twenty-nine drugs from hepatic microsomal intrinsic clearance data: an examination of in vitro half-life approach and nonspecific binding to microsomes. Drug Metab. Disp. 1999, 27, 1350-1359.

13. Chang, G.; Steyn, S. J.; Umland, J. P.; Scott, D. O. Strategic Use of Plasma and Microsome Binding To Exploit in Vitro Clearance in Early Drug Discovery. ACS Med. Chem. Lett. 2010, 1, 50-63.

14. Besnard, J.; Ruda, G. F.; Setola, V.; Abecassis, K.; Rodriguez, R. M.; Huang, X. P.; Norval, S.; Sassano, M. F.; Shin, A. I.; Webster, L. A.; Simeons, F. R.; Sojanovsky, L.; Prat, A.; Seidah, N. G.; Constam, D. B.; Bickerton, G. R.; Read, K. D.; Wetsel, W. C.; Gilbert, I. H.; Roth, B. L.; Hopkins, A. L. Automated design of ligands to polypharmacological profiles. Nature 2012, 492, 215-220.

15. Bridges, T. M.; Morrison, R. D.; Byers, F. W.; Luo, S.; Daniels, J. S. Use of a novel rapid and resourceefficient cassette dosing approach to determine the pharmacokinetics and CNS distribution of small molecule 7-transmembrane receptor allosteric modulators in rat. Pharmacol. Res. Perspect. 2014, 2, e00077.

16. Smith, N. F.; Raynaud, F. I.; Workman, P. The application of cassette dosing for pharmacokinetic screening in small-molecule cancer drug discovery. Mol. Cancer Ther. 2007, 6, 428-440. 
